\title{
PARDISC: A Cost Effective Model for Parallel and Distributed Computing
}

\author{
ACHUTHA RAMAN R \\ RAJKUMAR \\ HARI PRAKASH G \\ Operating System Group \\ Centre for Development of Advanced Computing \\ 2/1, Ramanashree Plaza, Brunton Road \\ Bangalore - 560 025, Karnataka, India \\ email: rajecdacb.ernet.in \\ BALA KISHORE B \\ BALA KISHOREB
}

Abstract

A homogeneous system of PCs, workstations, minicomputers, etc. connected together via a local area network or wide area network represents a large pool of computational power. However, in a network of PCs and Workstations transparency is not provided, and hence, users are aware of other machines. PARDISC is a parallel programming environment, which provides the needed transparency as a scalable OpenFrame Computing Model. PARDISC stands for PARallel and DIStributed Computing on homogeneous network. It supports three models of computing by providing the functionalities required to view any homogeneous network as a Loosely Coupled Parallel Computer, or Processor Pool Architecture, or Cluster of Workstations.

PARDISC aims at providing a cost effective parallel and distributed programming environment to the academic and $\mathrm{R}$ \& D institutions since, it employs the existing well established Local Area Network (LAN) network and models it to support both the paradigms. This paper presents an overview, design and architecture, which discusses how PARDISC can be used to configure the network as loosely coupled parallel machine, processor pool architecture, and distributed computing environment with Logical Network Connectivity. Software architecture discusses configuration servers, client processes, and processor pool servers and process communication interface of PARDISC. We end the paper with a description of some issues involved in its implementation on UNIX platform, and porting guidelines and its suitability for parallel programming.

Keywords: PARDISC, Parallel, Distributed, Processor Pool, Network Connectivity, Homogeneous Network, Configuration, Process Communication Interface.

\section{Introduction}

High speed network and improved microprocessor performance are making networks of workstations an appealing vehicle for parallel computing [5]. By relying solely on commodity hardware and software, network of workstations can

offer parallel processing at low cost. A network of workstations multiprocessor can be realized as a processor bank in which dedicated processors provide computing cycles, or it can consist of dynamically varying set of machines that perform long running computations during idle periods. In this case, the hardware cost is essentially zero, since many organizations already have extensive workstation networks.

In terms of performance, network of workstations approach or exceed supercomputing performance for some applications. The problem of locating and efficiently utilizing the resources in a network is an important factor [11]. PARDISC is designed to allow users to utilize the computing power of the network for executing user tasks, thereby providing them with an access to computational resources far beyond that provided by a standard system.

Several working systems $[2,4,7]$ have been built which offers some type of distributed computation. In [9], a system has been described based on Butler system which allows the users to execute jobs on remote workstations. It used a central idle machine registry to find a candidate idle machine. A distributed operating system, MACH-1 based on workstation model has been described in [3]. It is a microkernel based operating system in which user programs can run remotely when a specific machine gets overloaded. Amoeba [13] is a distributed operating system based on the processor pool concept. It makes a collection of CPUs and I/O devices act like a single computer. The PARAM 9000 Supercomputer supports both the Massively Parallel Processing (MPP) and Cluster Computing personalities [8]. In the MPP personality, compute nodes are loaded with PARAS microkernel and system servers, and service nodes are loaded with Solaris (Sun OS). Whereas, in the alternate personality all the nodes are configured as service nodes.

PARDISC provides a software model of integrated solution that is not found in a single unit in the earlier systems without any changes in the underlying current setup including network and operating system. 


\section{PARDISC at a Glance}

The main aim of this work is to build a transparent distributed environment, which is flexible, cost effective, and simpler parallel environment. PARDISC is a software model which provides the best of both worlds, Parallel and Distributed. It uses the existing network of homogeneous computers to run parallel programs using logical connectivity method and to execute distributed programs utilizing the power of other idle computers and pool processors in the network. The basic idea is to provide the users with the illusion of a single powerful time-sharing system, when, in fact the system is implemented on a collection of machines, potentially distributed across the network. The model of the PARDISC is depicted in Figure 1. for parallel and distributed applications. As a DCE, PARDISC provides a rich set of commands such as PARCPL (Parallel Compile), PARRUN (Parallel Run), PARMAKE (Parallel Make) etc., to distribute user tasks across the network transparently.

The network can be configured into any of the two modes, parallel or distributed or both, with the usage of two different configuration files. Each node maintains a per-process node connectivity status. It enables to identify neighborhood nodes. The configuration server maintains the global perprocess connectivity information. The interprocess communication between processes in and across processors is provided by Process Communication Interface (PCI).

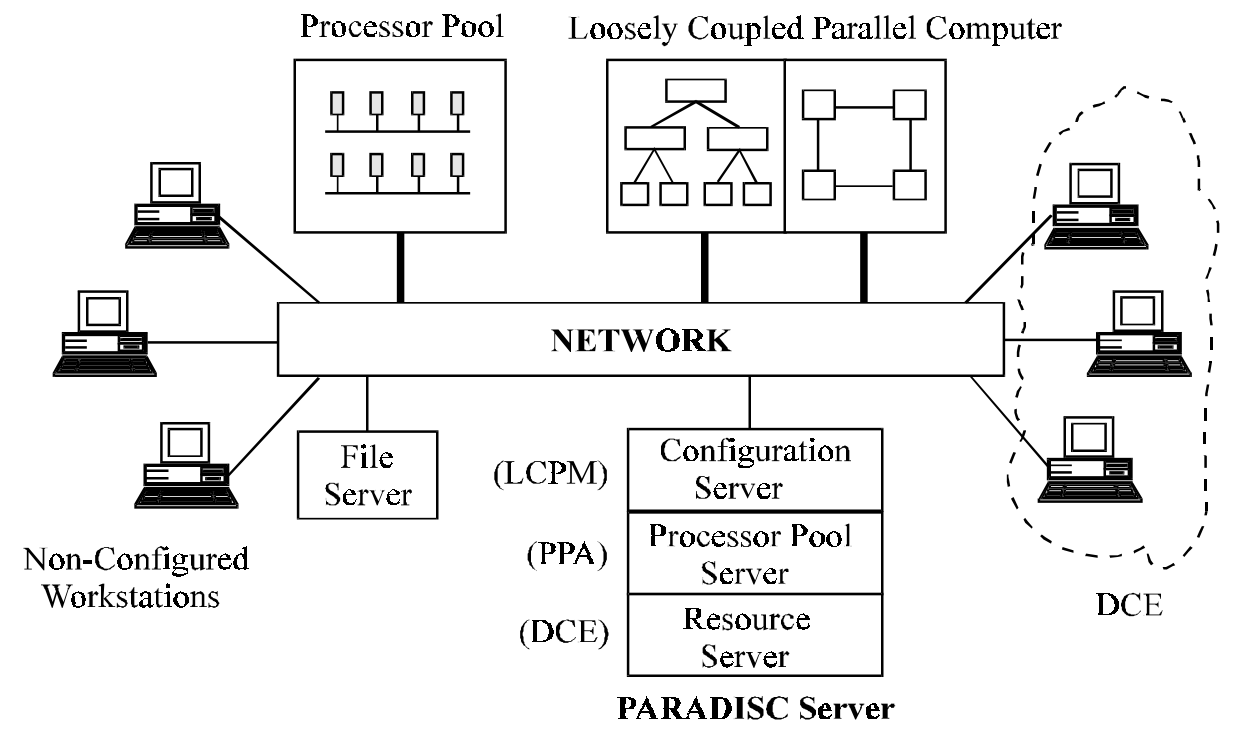

Figure 1: Architectural Model of PARDISC

PARDISC is typified by specialized components such as PARDISC server, which includes configuration server, resource server, and processor pool server. In each of the three configuration supported by PARDISC viz. Loosely Coupled Parallel Machine (LCPM) [6], Processor Pool Architecture (PPA) [1], and Distributed Computing Environment (DCE), it has a central server, running on one of the designated nodes (computers with main processor and memory), coordinating with loader servers, running on each of the other nodes, specific to the configuration.

PARDISC server configures the group of computers logically to the required topology and it allocates idle machines and pool processors for the users on demand. As a LCPM, the network can be configured to standard topologies such as tree, mesh, cube etc., or user defined topologies suitable to the problem domain. These topologies are logically connected through configuration server, provided by the PARDISC. As a PPA, user can make use of dedicated nodes
PCI provides two modes of communication: point-to-point and collective communication (broadcast and multicast), which are layered over the basic primitives such as send () and receive().

\section{Software Architecture}

The network can be configured in three aforementioned architectures by using different system configuration files. It contains the following information about the network configuration:

- number of nodes in the network,

- number of Processor Pool/DCE/LCPM nodes,

- maximum number of tasks that can be loaded per node,

- resources available on each node,

- default specification, etc.

The PARDISC server provides three different functional services depending on the architecture. It works as Configu- 
ration Server in case of LCPM, Processor Pool Server in case of PPA, and as a Resource Server in case of DCE architecture. All other nodes except PARDISC server node register their network addresses and ports with the PARDISC server at boot time.

\section{Loosely Coupled Parallel Machine (LCPM)}

The parallel application can be structured as follows:

- As a set of application-level instruction sequence, we call subtasks.

- As a set of processing module that executes subtasks.

- As a master module (root), generates new subtasks based on the result of the other subtasks and detects sufficient condition for termination.

The application can use different processors for the execution of subtasks. Each processor executes one subtasks and passes the output to the master task, which then redirects the final results to the client machine from which the user is interacting. LCPM configuration is best suitable for such kind of problem domains.

The components of LCPM model are configuration server, load server, and PARDISC loader. (See Figure 2) A configuration server, which runs on a single dedicated machine, is responsible for establishing the logical connection among the other nodes according to the defined topology (tree, mesh, cube, etc., or user defined topologies). A load server runs on each of the other nodes (except configuration sever node) and is responsible for (loading and) spawning of tasks. PARDISC loader is the front end user interface, which loads the user tasks on remote nodes, assigned by the configuration server.

\section{Nodes configured as tree topology}

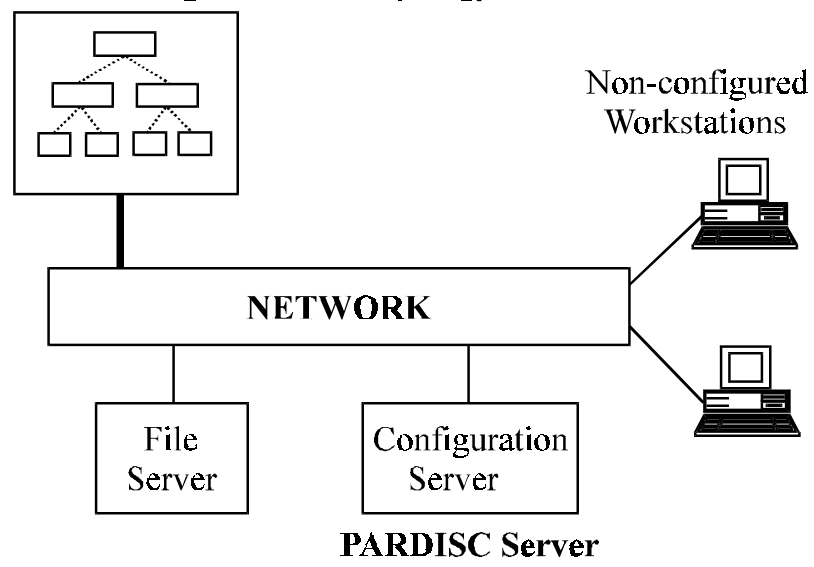

Figure 2: Loosely Coupled Parallel Machine

A configuration file allows the user to specify any topology in LCPM. It consists of configuration and loading sections. Configuration section specifies the topology and the relation of each node with its neighborhood nodes and loading section specifies the placement of tasks on nodes. The typical format of configuration file is as follows:

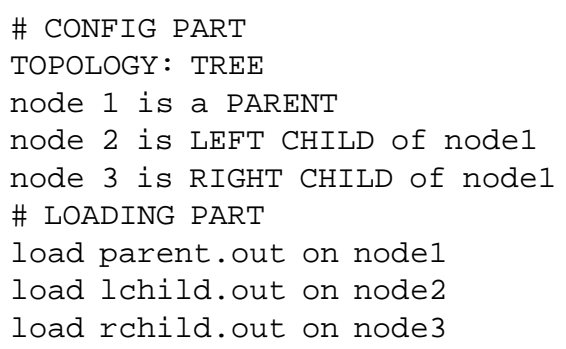

The node1 refers to the root (may be the node from where the job has been submitted) whereas, the node $2, .$. , nodeN are other nodes of the topology.

PARDISC loader sends the configuration information to the configuration server, which allocates requested number of nodes (by PARDISC loader) by accessing its internal resource table and creates the topology table. Typically, the topology table contains the following information:

- topology name

- role of each node in the topology (e.g., PARENT, CHILD, etc.).

- immediate neighbors and their relation to one another.

- group id and node ids of all the nodes in the topology.

- originator id (root node id)

Configuration server sends the ALLOCATE_REQ message to all the load servers running on the allocated nodes for the current job. In response to this message all the node servers create communication port to load the tasks and registers the same with the configuration server. Configuration server updates its (current group) topology table with the communication ports, and sends this table to all the load servers of the current job and to the PARDISC loader. PARDISC loader downloads the user tasks to the load server communication ports of the configured nodes according to the user specified configuration.

Once the tasks are loaded on the respective nodes, they start executing and the tasks can communicate with other tasks of its topology using PCI calls. (Unlike any message passing interface, PCI allows to use logical node identifier like PARENT, CHILD, etc. which are specified in configuration file.) Since every tasks has the topology table (shares with load server), any one can communicate with its related neighbor within their topology. On complete execution of the job, PARDISC loader informs the configuration server to release the resources allocated for the job.

\section{Processor Pool Architecture (PPA)}

In PPA, all the computing power of a network is located in one processor pool, which consists of multiple CPUs each with its local memory and network connections. Users can be assigned as many CPUs as they need for short periods by the Processor Pool (PP) server after which they are returned 
to the pool, so that other users can make use of them. There is no concept of ownership here; all the processors belong equally to everyone. Many parallel and concurrent applications performance may not be satisfactory, if they scheduled by traditional time-sharing systems, but with dedicated processors some parallel applications performance are improved. Processor pool model is the best suited for this kind of applications. The PPA of PARADISC is shown in Figure 3.

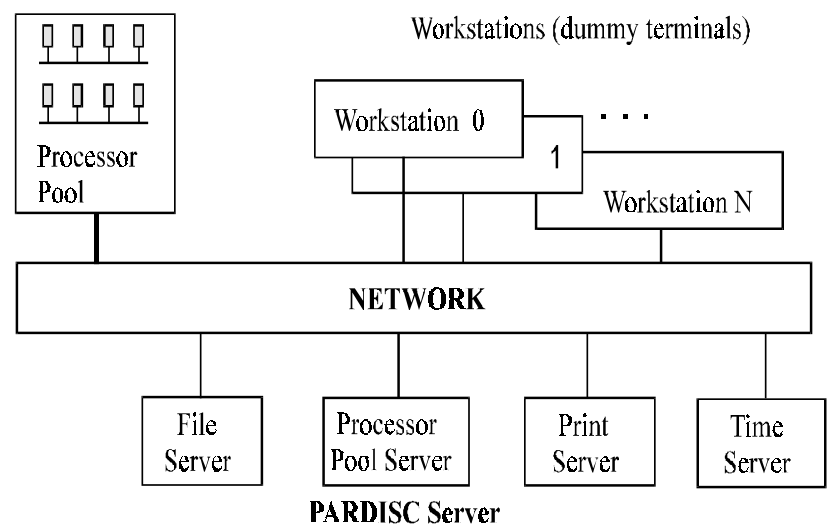

Figure 3: Processor Pool Architecture of PARDISC

In a LAN each node has a processor and local memory. A group of such nodes collectively form the Processor Pool. The nodes which are not part of the pool act as client machines. User logs into the system only through the client machines, which are used as dummy terminals. Processor Pool server runs on a designated node to manage the pool processors. User submits tasks to the client machine through the PARDISC loader, which in turn requests the PP server to allocate require number of nodes. PP server allocates the requested number of processors form the pool and returns the corresponding node-ids and port-ids of the load server to the client. The client after acquiring the node-ids, starts downloading the user tasks to the appropriate nodes. Load server spawns the tasks on its node and redirects the results to the owner machine. Finally, on completion of the tasks, client requests the PP server to release the allocated processors and they are returned to the pool. The PP nodes can also be configured for LCPM model.

\section{Distributed Computing Environment (DCE)}

In DCE, each machine retains its own identity. i.e., user can log into any machine and can access any other machine in the network. Each node is a separate machine as in the case of LCPM, but distributed tasks can be executed transparently across the network on lightly loaded machines, which is managed by the Resource Server. (See Figure 4.) It gives the single system image for the user so that user tasks can be compiled, loaded and executed any where on the network. The results of the task are redirected to the user owned machine.

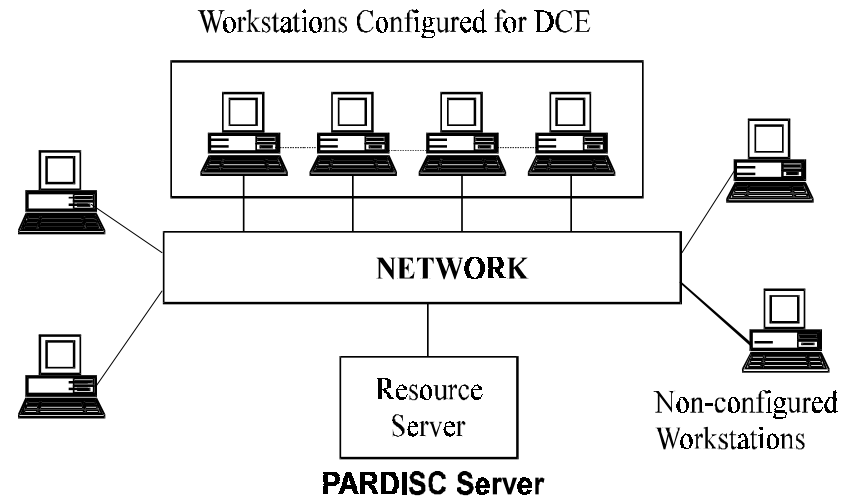

Figure 4: Distrubuted Computing Environment of PARDISC

In DCE, user views the network as a loosely coupled cluster of computers. A configuration file specifies which tasks should be loaded on which logical nodes. In general, user views DCE as a virtual machine. Resource Server in this case is responsible for assigning the nodes for the user tasks uniformly across the network. It maintains processor-load table which maintains the most recent information of the load of all the DCE processors. This table is periodically updated by the load servers running on the DCE machines. So that it can assign lightly loaded machines evenly when resources are requested.

\section{Process Allocation and Synchronization}

PARDISC server is responsible for process allocation in all the above discussed models. It implements gang scheduling, which guarantees simultaneous scheduling of parallel applications over multiple processors on the network. This implies that, processor allocation should be done for any parallel application, only if all the tasks of a parallel application can be loaded on the allocated nodes without fail. The PARDISC server collects processor health status over a periodic interval of time from all the processors. If any one of the allocated processor to an application happen to be dead at any point of time, then all tasks of that application has to be aborted. PARDISC server does this monitoring job, and in case of error conditions it aborts the application and sends the error notification message to the PARDISC loader.

The processor allocation is also based on priority. For example, interactive jobs can be treated as high priority and the batch jobs can be assigned low priorities. Priority assignment are configurable. For example, priorities can be classified based on user groups, type of jobs, and other user specified parameters. Default processor allocation statergy is also available for PPA and DCE models. If the number of processors or loading configuration information is not specified by the user, then PARDISC server allocates processors less than or equal to the default number of processors, which is given in the system configuration file. 


\section{User Interface}

To give the required transparency to the users, PARDISC implements a set of user interfaces for load, control, and monitor user applications.

In all the three models, pload (PARDISC loader) is supported to load the parallel and distributed applications on PARDISC environment. Pload takes the configuration file as the input. In LCPM, configuration file contains two part, config and loading parts, whereas in the other two models, the configuration file contains only the loading part. DCE and PPA supports a rich set of commands such as PARCPL (Parallel Compile), PARRUN (Parallel Run), PARMAKE (Parallel Make), etc. to provide location transparent execution of the user jobs (set of tasks). PARCPL transfers independent source files to different nodes, which compiles and links the same on those remote nodes and transfers the executable to the owner machine. PARMAKE is used to build executable from the multiple source files of the project. It is performed by transferring each source file to a different node of DCE/PPA and compiles the same on those remote nodes and transfers the object files to the owner machine, which are in turn linked to produce an executable on owner machine. PARRUN distributes user tasks across the network for execution.

Job control commands are supported to display the job/ task status, to suspend, to resume, and to kill the jobs or tasks. Job statistics commands to collect the statistics of any application over the network and monitoring commands to trace the job activities, processor load and other information are also supported.

\section{Process Communication Interface}

Process Communication Interface (PCI) is a message passing interface of PARDISC supporting communication among tasks in the loosely coupled parallel machine configuration. The Application Program Interface (API) calls supported by PCI are consistent irrespective of device specific communication protocols and hence provide the portability feature to the PCI users. The functionality of the PCI is designed to provide simpler and flexible syntax, and is based on current common practice, and is similar to that provided by widely used message passing systems such as PVM [12], MPI [14], etc. Unlike PVM and MPI interfaces, PCI interface calls are particularly designed for PARDISC environment, where the communication among the tasks are expressed using logical node connectivity. API calls supported by PCI fall into following three categories.

- Point-to-Point Communication,

- Group Communication, and

- Signal Passing.

All these interfaces are layered on top of basic primitives PCI_Send() and PCI_Receive(). PCI supports both synchronous (DELAY) and asynchronous (NO_DELAY) modes of communication. The syntax of some of the important PCI calls are listed below:

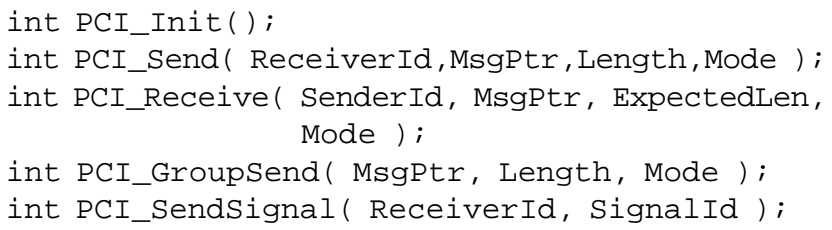

The logical task-ids used with PCI calls are the same as those specified in the configuration file. The mapping of logical-ids to the physical address is performed by the PCI library with the help of per-group port topology table and it is maintained by each tasks. PCI_Init () creates two ports, one for sending the message and the other for receiving the message, and registers them with root (master) process (which is assigned by the PARSISC loader) of its group, which then builds the group port table of that group, and multi-casts the table to the group members. With this, every task can communicate with every other tasks of that group independently.

To support asynchronous communication, PCI library maintains inbound queues to store the unposted receive messages. The group communication calls support communication among the tasks of the same group (group-cast). Signals can also be passed between tasks. Signals are send to the load server of the destination node and it is delivered to the target task, as local signal from the load server.

\section{Implementation Issues}

The implementation of PARDISC version 1.0 is initiated on a network of computers running SVR4 Unix operating system. It aims at source-level compatibility across SVR4 Unix systems. It uses PCI as the message passing library using TCP/IP sockets as a low level device specific protocol. A multithreaded implementation of the PARDISC on multithreaded operating systems, such as Solaris and Mach, improves the performance.

\section{Conclusions}

Since PARDISC supports parallel and distributed computing with the existing network of computers, it can be used as a developing and testing environment for the parallel and distributed programs. In LCPM configuration, distributed parallel algorithms like distributed sorting, etc., can be developed and tested. Using DCE configuration, distributed algorithms like clock synchronization algorithm, election algorithms, etc., can be developed and tested. In PPA, both parallel and distributed algorithms can be performed more efficiently using the power of dedicated processors.

In LCPM mode of PARDISC, the programmer must divide the computation among different tasks and use message passing facilities of PCI to control interaction among the concur- 
rent tasks. It is suitable for both the data and process parallelism [10]. Programmer need not worry about where the tasks are loaded physically and how to communicate with them.

The three personalities of high performance computing, LCPM, PPA, and DCE are integrated into the PARDISC to realize a cost effective solution with zero extra hardware and no modification to the operating system.

\section{Technical Support}

We would be happy to provide technical support for the implementors of PARDISC on various computing platforms. Enquiries on implementation of PARDISC on heterogeneous platform are welcome.

\section{Acknowledgments}

The authors are grateful to all the members of C-DAC, Bangalore, and in particular all the members of Operating System Group. We owe a debt of gratitude to Prof. Vishwanathan Iyer K, Regional Engineering College, Tiruchirapalli, and Prof. Venugopal K R and Ms. Mangala (Radha), University Visvesvaraya College of Engineering, Bangalore University for their useful conversations and comments during the implementation and an earlier draft of the paper.

\section{References}

1. Achutha Raman R, Remote Task Execution In Processor Pool Environment, M. Tech. Thesis, Regional Engineering College, Tiruchirapalli, India, 1994.

2. Ashfaq A, Viktor K Prasana, Muhamad, and Cho-hi Wang, Hetrogeneous Computing: Challenges and Opportunities, IEEE Computer, June 1993.

3. Baron R, et al., MACH-1: An operating environment for large scale multiprocessor applications, IEEE Software, Vol. 2, 1985.

4. Cheriton D R, The V Distributed System, Communications of ACM, Vol 31, 1988.

5. Christiane Amza et. al., ThreadMarks: Shared Memory Computing on Network of Workstations, IEEE Computer, February 1996.

6. Hari Prakash G, Loosely Coupled Parallel Architecture on a LAN, M. Tech. Thesis, Regional Engineering College, Tiruchirapalli, India, 1994.

7. Henry Clark and McMillan B, DAWGS - A Distributed Computer Server Utilizing Idle Workstations, Journal of Parallel and Distributed Computing, Vol. 14, 1992.

8. Mohan Ram N et al., The Operating Environment, The PARAM 9000, (c) C-DAC, 1996.

9. Nichols D A, Using Idle Workstations in a Shared Computing Environment, Operating System Review, November 1987.

10. Rajkumar et al., PEACE Threads Interface on Microkernel, Proceedings of The Third Conference On Advanced Computing, Tata McGraw Hill, India, 1995.
11. Spector A Z, Performing Remote Operations Efficiently on a LAN, Communications of ACM, Vol 24(4), 1982.

12. Sunderam V S, PVM: A Framework for Parallel and Distributed Computing, Journal of Concurrency: Practice and Experience, Vol 2(4), 1990

13. Tanenbaum A S, et al, Amoeba: A Distributed Operating System for the 1990s, IEEE Computers, Vol 23, 1990.

14. The MPI Forum, MPI: A Message Passing Interface Standard, May 1994.

\section{About the Authors}

Achutaraman $\boldsymbol{R}$ is a Software Engineer, HP-Indian Operations, Bangalore. He has completed Bachelor of Engineering in Electronics and Instrumentation from the Annamalai University in the year 1993 and Master of Engineering in Computer Science from the Bharathidasan University in the year 1994. His research interests include Distributed Computing, Networking, and Parallel Algorithms. He can be reached by e-mail at achu@india.hp.com.

Rajkumar is a Member of the Technical Staff, Operating Systems Group, C-DAC, Bangalore. He received the Bachelor of Engineering degree in Computer Science from the University of Mysore in the year 1992 and the Master of Engineering degree in Computer Science from the University of Bangalore in the year 1995. He was awarded the Dharma Ratnakara Memorial Trust Gold Medal for securing the I Rank in the year 1992. He has coauthored the books, Microprocessor x86 Programming and Object Computing with $\mathrm{C}++$. Besides being a visiting faculty for the Bangalore University, he is conducting a popular course on the Java by Email which is circulated around the globe. His research papers in the area of High Performance Computing have appeared in National and International Conferences. His research interests include Graphics, Programming Paradigms, Multithreaded Computing, and Microkernel OS for MPP. He can be reached by e-mail at raj@ @ cdacb.ernet.in.

Hari Prakash $\boldsymbol{G}$ is a Senior Software Engineer, HCL-HP, Madras. He has completed Bachelor of Engineering in Computer Science in the year 1992 and Master of Engineering in Computer Science, both from the Bharathidasan University in the year 1994. His research interests include Distributed Computing, Genetic Algorithms, and Parallel Algorithms. He can be reached by e-mail at ghari@ $@$ hclt.com.

Bala Kishore B is a Software Engineer, Silicon Automation Systems, Bangalore. He has completed Bachelor of Engineering in Electronics from the Osmania University in the year 1993 and Master of Technology in Computer Science from the Kakatiya University in the year 1995. His research interests include Real Time Systems and Operating Systems. He can be reached by e-mail at bala@sas.soft.net. 